\title{
Lambda sign on a gallium scan: not always sarcoidosis
}

\author{
Evgenia I. Kalamara ${ }^{1}$, Evangelos T. Ballas ${ }^{2}$, Evangelia S. Panagiotidou ${ }^{3}$ \\ ${ }^{1}$ Sotiria Regional Chest Disease Hospital of Athens; '2Cardiology Clinic, General Hospital of Athens "G. Gennimatas", \\ Athens; ${ }^{3}$ Respiratory Failure Clinic, General Hospital of Thessaloniki "G. Papanikolaou", Thessaloniki, Greece
}

\begin{abstract}
Sarcoidosis is a systemic disease of unknown origin. We describe a case of sputum smear-and culture-negative tuberculosis that was diagnosed with histological examination of a surgical lung biopsy, as other entities such as sarcoidosis could not be excluded after extended investigation. Even a typical lambda sign on gallium scintigraphy proved to be misleading.
\end{abstract}

\section{Introduction}

Tuberculosis (TB) is an infectious granulomatous disease that can affect the lungs, as well as other organs. Despite the medical

Correspondence: Evgenia I. Kalamara, Pavlou Mela 3, Chortiatis, 57010 Thessaloniki, Greece.

Tel. +30.6948107468 .

E-mail: gina.kal88@gmail.com

Key words: tuberculosis; sarcoidosis; culture-negative; lambda sign.

Authors' contributions: EIK, participated actively in the diagnostic procedure of the patient, acquired informed consent and wrote the main part of the manuscript; ETB, reviewed current literature and contributed to writing; ESP, reviewed the manuscript and current literature and contributed to writing. All authors have read and approved the final manuscript. All authors agree to be accountable for all aspects of the work in ensuring that questions related to the accuracy or integrity of any part of the work are appropriately investigated and resolved.

Conflict of interests: the authors declare no conflict of interest.

Informed consent: Written informed consent was acquired from the patient for the publication of this study.

Ethical approval: Ethical approval was not acquired, since this manuscript is a case report and was not produced in the context of a research trial. Informed consent was considered sufficient.

Received for publication: 7 February 2020

Accepted for publication: 25 March 2020.

${ }^{\circ}$ Copyright: the Author(s), 2020

Licensee PAGEPress, Italy

Monaldi Archives for Chest Disease 2020; 90:1247

doi: 10.4081/monaldi.2020.1247

This article is distributed under the terms of the Creative Commons Attribution Noncommercial License (by-nc 4.0) which permits any noncommercial use, distribution, and reproduction in any medium, provided the original author(s) and source are credited. advances so far it remains one of the deadliest infections worldwide [1]. Sarcoidosis is also a granulomatous disease of unknown etiology that meets the criteria for multi-organ disease [2]. In some cases, the clinical manifestations of these two diseases overlap, creating a diagnostic challenge.

The current gold standard for the diagnosis of sarcoidosis remains tissue histological examination. However, other methods can aid the diagnostic process, such as gallium 67 (67Ga) scintigraphy. The characteristic panda and lambda signs may be indicative of the disease, although rarely they can be found in different clinical situations.

\section{Case Report}

A male Caucasian 39-year-old patient presented in the emergency department complaining of dry cough and a reddish, painful, tender rash below the knees. He had an arterial pressure of 110/70 $\mathrm{mmHg}$, a temperature of $36.6^{\circ} \mathrm{C}$, a respiratory rate of 18 breaths/min, and a normal oxygen saturation. Lung sounds were normal on auscultation and clinical examination revealed no pathological findings except for erythema nodosum in the lower extremities. His medical history was unremarkable, apart from rheumatic fever during childhood. The patient was a non-smoker and reported no alcohol consumption. His occupation was professional driving.

Laboratory examinations were within normal limits, except for a high sedimentation rate of $45 \mathrm{~mm}$. On presentation, the chest radiograph detected enlarged portal lymph nodes bilaterally, as well as diffuses alveolar infiltrates located mainly in the right lower lung zones (Figure 1).

The spectrum of differential diagnosis based on clinical presentation and bilateral portal lymph node enlargement included:

1. Sarcoidosis;

2. Infections (tuberculosis, nontuberculous mycobacteria, coccidioidomycosis);

3. Malignancies (lymphoma, metastatic carcinoma);

4. Occupational diseases (silicosis, berylliosis).

Subsequently, a chest computed tomography was ordered. The findings were enlarged mediastinal lymph nodes bilaterally, interlobular septal thickening, perilymphatic nodules, as well as multiple nodules with random distribution (Figure 2). Further laboratory evaluation revealed a high serum ACE (70IU/L), normal $24 \mathrm{~h}$ urine calcium levels and a normal protein electrophoresis test. Sputum cultures for common pathogens and sputum cytology were unremarkable. A full investigation for tuberculosis was performed: a positive Tuberculin Skin Test $(20 \mathrm{~mm})$ was noted, while sputum Acid Fast Bacilli (AFB) smear, AFB culture and Nucleic Acid Amplification Testing were negative. Testing for HIV infection was negative.

Pulmonary function testing was within normal range: FVC 


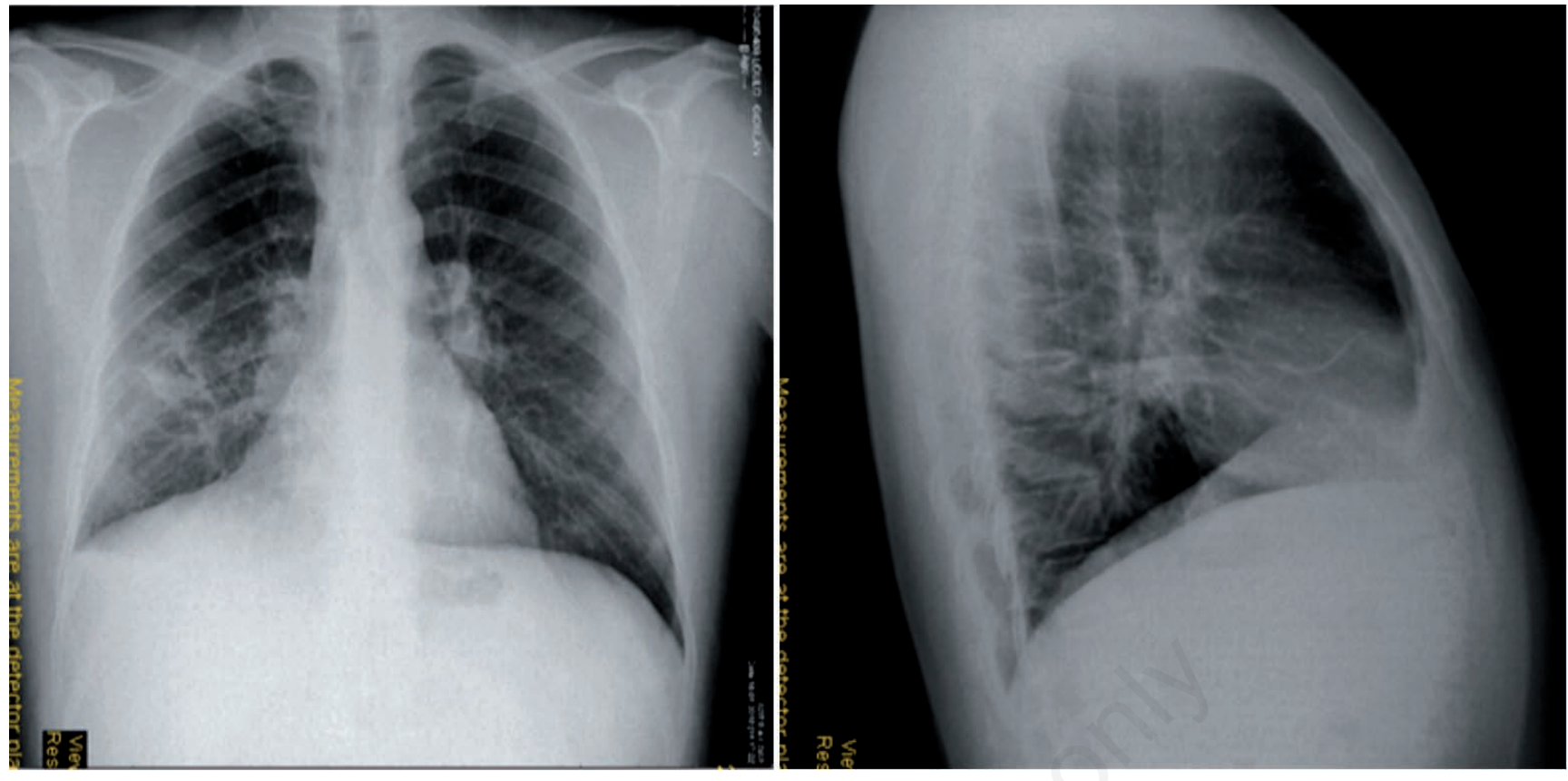

Figure 1. Chest radiograph on presentation (left, posteroanterior view; right, lateral view).
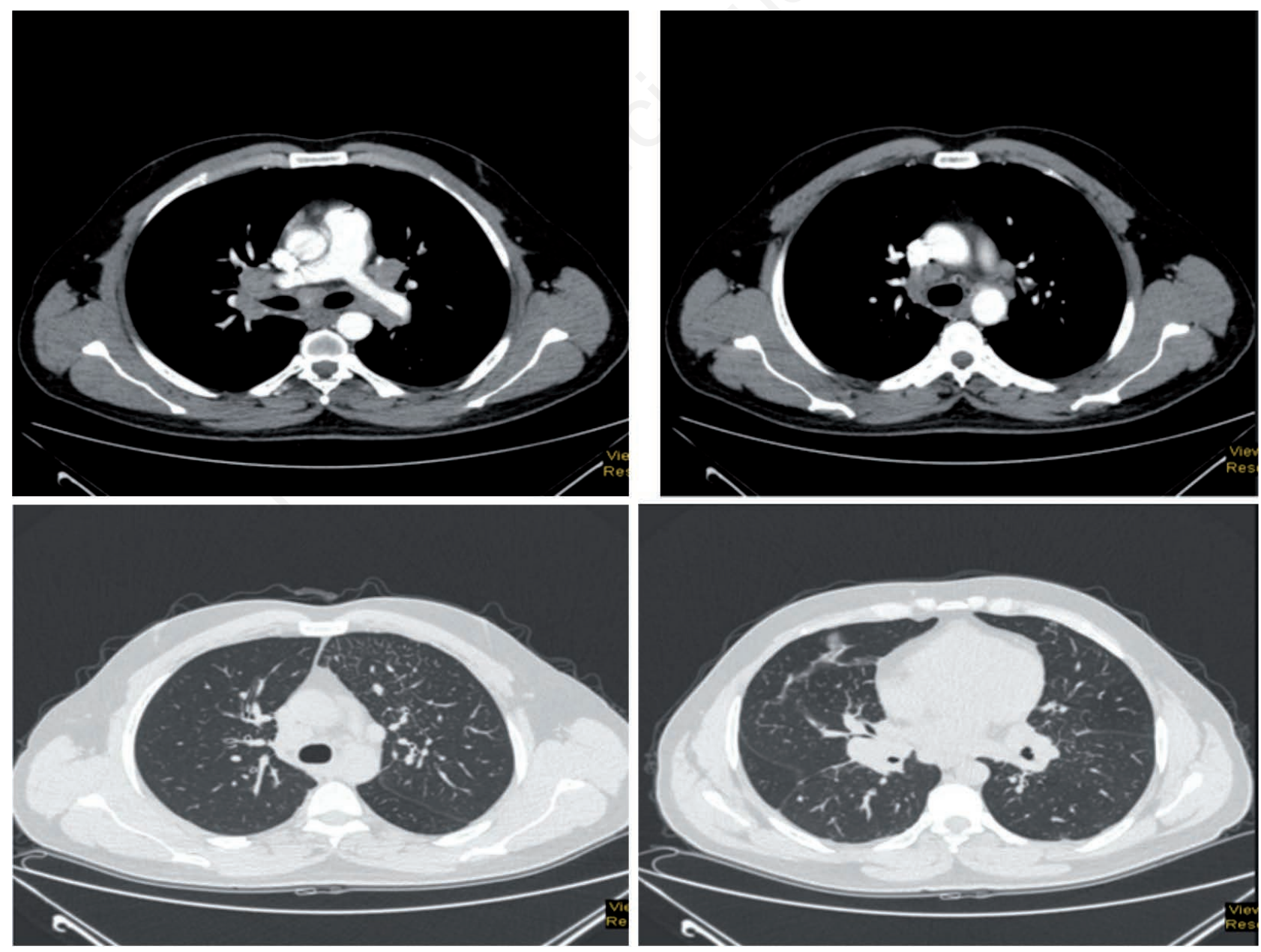

Figure 2. Chest CT showing mediastinal lymph nodes bilaterally, interlobular septal thickening, perilymphatic nodules and multiple nodules with random distribution. 
$4.490 \mathrm{ml}$ (93\% pred), FEV1: $3.310 \mathrm{ml}$ (83\% pred), FEV/FVC 0,73, DLCO: $89 \%$ pred.

The next diagnostic step was bronchoscopy with bronchoalveolar lavage (BAL). Macroscopically, the appearance of the tracheobronchial tree was normal. The BAL sample had an eosinophilic cellular pattern (macrophages 80,4\%, lymphocytes $13,4 \%$, eosinophils $3 \%$, neutrophils $3,1 \%$ ). Cytology, AFB smear, AFB cultures, cultures for fungi and for common pathogens from bronchial washing fluid samples were negative.

A gallium-67 scan was ordered, as sarcoidosis was considered the most probable diagnosis. It revealed a characteristic Lambda sign (Figure 3). However, none of the above investigations was certainly diagnostic. A surgical lung biopsy was then performed, which revealed epithelioid necrotic granulomas and the presence of multinucleated giant cells. Molecular testing and AFB cultures from biopsy specimens were positive. The findings confirmed the diagnosis of tuberculosis.

\section{Discussion}

Tuberculosis has always been a diagnostic and therapeutic challenge for clinicians. The spectrum of its manifestations is vast, the recalcitrance to eradication is remarkable and unfortunately sputum cultures are not always reliable to exclude the disease.

Sputum culture-negative tuberculosis is not a rare problem. It is estimated that approximately $17 \%$ of newly diagnosed cases are culture-negative [3]. Usually these patients present with fewer symptoms and less cavitation on imaging than patients with cul- ture-positive TB [4]. The 2003 joint TB treatment guideline by the American Thoracic Society (ATS), Centers for Disease Control and Prevention (CDC), and Infectious Diseases Society of America (IDSA) states that patients highly suspicious for active TB (at-risk groups, abnormal chest radiograph, positive TST, no other diagnosis confirmed) should receive multidrug therapy for active TB, even if there is no bacteriological confirmation of the infection [5].

Sarcoidosis is a clinical entity that can be sometimes misdiagnosed as TB and vice versa. Interestingly, Mycobacterium tuberculosis has been extensively studied as a possible cause of sarcoidosis [6]. Sarcoidosis is a much rarer disease, its incidence is estimated at around 16.5/100,000 in men and 19/100,000 in women [7].

Symptoms like cough, malaise, fever, weight loss, erythema nodosum can appear in both diseases, and both can involve multiple organs. Histologically, both TB and sarcoidosis are granulomatous. However, TB is characterized by caseating and sarcoidosis by noncaseating granulomas. This differentiation is cardinal. Besides, the diagnosis of sarcoidosis relies on the clinical and radiographical presentation and evidence of noncaseating granulomas on biopsy specimens after other granulomatous disorders have been excluded [8]. Furthermore, chest imaging in both diseases can be similar (mediastinal and hilar lymphadenopathy, multinodular pattern, consolidation). In this case, gallium-67 scan results were also misleadingly typical of sarcoidosis. Table 1 compares and summarizes the main features of TB and sarcoidosis [9].

Gallium-67 $(67 \mathrm{Ga})$ acts like an iron analogue and binds to transferrin. In inflammatory lesions, $67 \mathrm{Ga}$ binds to lactoferrin, a protein in polymorphonuclear leukocytes [10]. The role of gallium-67 scintigraphy for the diagnosis of sarcoidosis has been extensively evaluated. In this case, its sensitivity ranges between

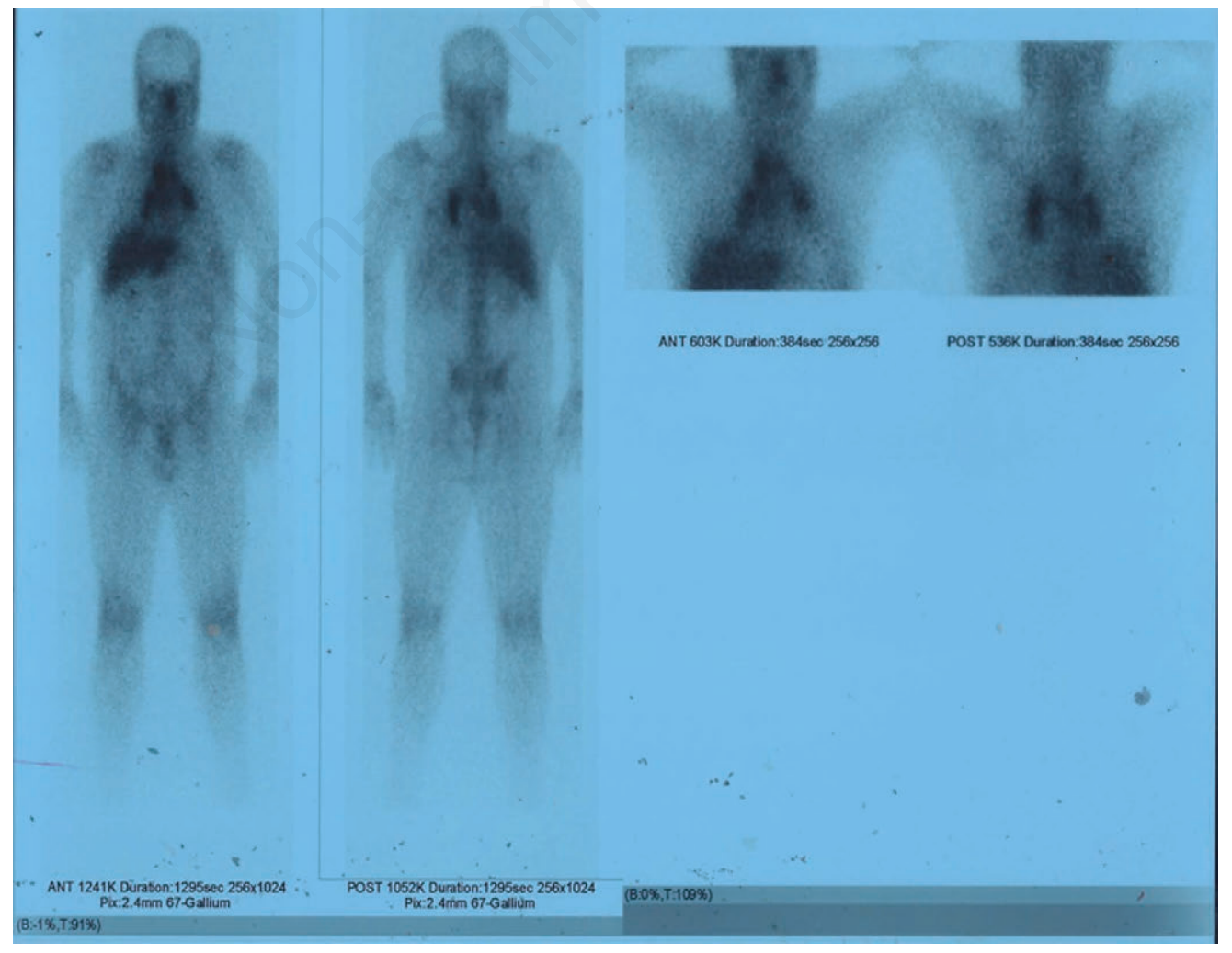

Figure 3. Gallium-67 scan. 
Table 1. Differentiating features in TB and sarcoidosis

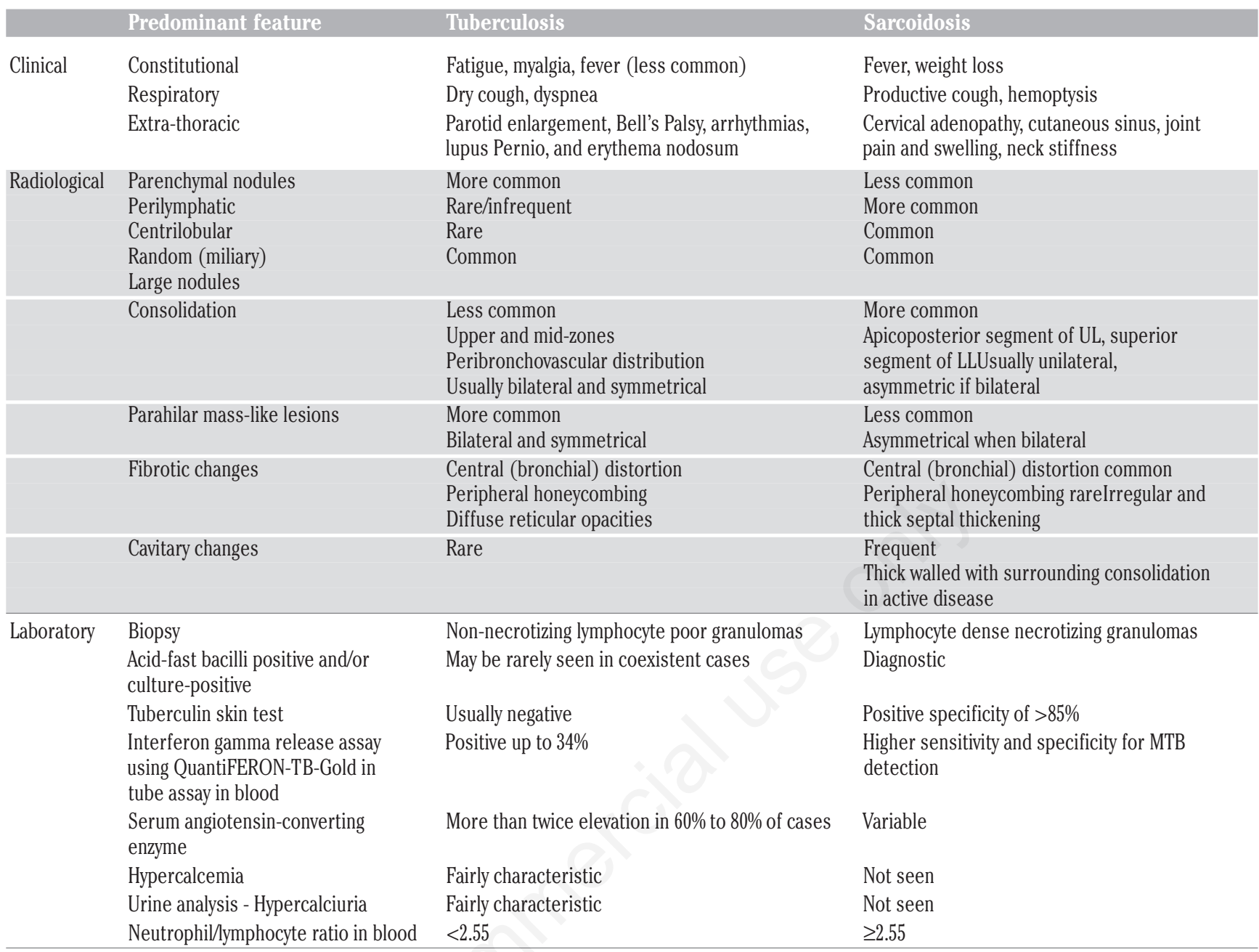

$60-90 \%$, with a low specificity of $\sim 50 \%$. On the other hand, negative $67 \mathrm{Ga}$ scintigraphy with normal angiotensin-converting enzyme (ACE) has a high negative predictive value.

The "lambda" and "panda" signs in 67Ga scintigraphy are considered typical of sarcoidosis. Active bilateral hilar and right sided mediastinal lymph nodes form the lambda sign. The panda sign represents activity in the lacrimal and parotid glands [11]. Simultaneous lambda and panda images are highly specific for sarcoidosis [12]. However, the panda sign can also be found in other conditions (HIV, lymphoma, Sjögren's syndrome, previous irradiation of the neck $[11,13]$. The Lambda sign can also rarely be seen in patients without sarcoidosis [13] but data referring to other possible diagnosis is scarce.

\section{Conclusions}

This case is an example of how complex it can be to diagnose tuberculosis, a disease very extensively studied that can mimic several other conditions and still eludes eradication. Even though sputum cultures are the cornerstone of diagnosis, the entity of smear-negative and culture-negative tuberculosis is not an uncom- mon occurrence. Alertness is always substantial, as even a typical sign of a disease can be attributed to another and misdiagnosis can lead to serious consequences, such as the spread of a potentially lethal infection.

\section{References}

1. Smith I. Mycobacterium tuberculosis Pathogenesis and molecular determinants of virulence. Clin Microbiol Rev 2003;16: 463-96.

2. Kummer F, Klech H. Sarcoidosis as a multi-organ disease. Pneumologie 1990;44:S158-61.

3. Hamada Y, Paulos L, Baruch NG, Cronin W. Proposed approach for 4-month treatment of culture-negative pulmonary tuberculosis in adults. Ann Am Thorac Soc 2016;13:1657-8.

4. Nguyen MVH, Levy NS, Ahuja SD, et al. Factors associated with sputum culture-negative vs culture-positive diagnosis of pulmonary tuberculosis. JAMA Netw Open 2019;2:e187617.

5. American Thoracic Society, CDC, Infectious Diseases Society of America. Treatment of tuberculosis. Morbidity and Mortality Weekly Report Recommendations and Reports. 
2003;52(RR-11):1-77. Erratum in: Morbidity and Mortality Weekly Report 2005;53(51\&52);1203.

6. Mortaz E, Masjedi MR, Abedini A, et al. Common features of tuberculosis and sarcoidosis. Int J Mycobact 2016;5:S240-1.

7. Nunes H, Bouvry D, Soler P, Valeyre D. Sarcoidosis. Orphanet J Rare Dis 2007;2:46.

8. [No authors listed]. Statement on sarcoidosis. Joint Statement of the American Thoracic Society (ATS), the European Respiratory Society (ERS) and the World Association of Sarcoidosis and Other Granulomatous Disorders (WASOG) adopted by the ATS Board of Directors and by the ERS Executive Committee, February 1999. Am J Respir Crit Care Med 1999;160:736-55.

9. Bhalla A, Das A, Naranje P, et al. Dilemma of diagnosing tho- racic sarcoidosis in tuberculosis endemic regions: An imagingbased approach. Part 1. Indian J Radiol Imaging 2017;27:369.

10. Weiner R, Hoffer PB, Thakur ML. Lactoferrin: its role as a Ga67-binding protein in polymorphonuclear leukocytes. J Nucl Med 1981;22:32-7.

11. Keijsers RGM, Van Den Heuve DAF, Grutters JC. Imaging the inflammatory activity of sarcoidosis. Eur Respir J 2013;41:743-51.

12. Sulavik SB, Spencer RP, Weed DA, et al. Recognition of distinctive patterns of gallium-67 distribution in sarcoidosis. J Nucl Med 1990;31:1909-14.

13. Yoshimizu T, Suga K, Orihashi N, et al. The appearance of "lambda" and "panda" sign on Ga-67 scintigraphy in sarcoidosis. Kaku Igaku 1991;28:1151-7. 